\title{
Synchronization of Spontaneous Eyeblink during Formula Car Driving
}

\author{
Ryota Nishizono \\ ryota.nishizono.kw@hco.ntt.co.jp \\ NTT Communication Science \\ Laboratories \\ Atsugi, Kanagawa, Japan
}

\author{
Naoki Saijo \\ naoki.saijo@hco.ntt.co.jp \\ NTT Communication Science \\ Laboratories \\ Atsugi, Kanagawa, Japan
}

\author{
Makio Kashino \\ makio.kashino.ft@hco.ntt.co.jp \\ NTT Communication Science \\ Laboratories \\ Atsugi, Kanagawa, Japan
}

\begin{abstract}
Formula car racing is a highly competitive sport. Previous studies have investigated the physiological characteristics and motor behaviors of drivers; however, little is known about how they modulate their cognitive states to improve their skills. Spontaneous eyeblink is a noteworthy factor because it reflects attentional states and is important for drivers to minimize the chance of losing critical visual information. In this study, we investigated whether the blink rate, blink synchronization among laps in each driver, and synchronization across drivers were related to their performance. Toward this end, we recorded the blinks and car behavior data of two professional drivers in quasi-racing environments. The results showed higher synchronization in higher-performance laps of each driver and across drivers but no significant change in blink rate. These results suggest that blink synchronization could reflect the changes in performance mode during formula car driving.
\end{abstract}

\section{CCS CONCEPTS}

- Human-centered computing $\rightarrow$ Empirical studies in HCI; - Computing methodologies $\rightarrow$ Activity recognition and understanding.

\section{KEYWORDS}

blink, eye metrics, car driving, motor sport, in-the-wild data

\section{ACM Reference Format:}

Ryota Nishizono, Naoki Saijo, and Makio Kashino. 2021. Synchronization of Spontaneous Eyeblink during Formula Car Driving. In ETRA '21: 2021 Symposium on Eye Tracking Research and Applications (ETRA '21 Short Papers), May 25-27, 2021, Virtual Event, Germany. ACM, New York, NY, USA, 6 pages. https://doi.org/10.1145/3448018.3458002

\section{INTRODUCTION}

Formula car racing is a very popular and challenging sport. Formula car racing includes various racing categories such as Formula One (F1), Formula Two (F2), and Formula Three (F3); various national leagues; Formula E; and the IndyCar Series. In this sport, car speeds can reach as high as $300 \mathrm{~km} / \mathrm{h}$, and drivers' victory margins can be in fractions of a second. Therefore, this sport is highly competitive,

\section{(cc) 980}

This work is licensed under a Creative Commons Attribution-NonCommercialShareAlike International 4.0 License.

ETRA '21 Short Papers, May 25-27, 2021, Virtual Event, Germany

(C) 2021 Copyright held by the owner/author(s).

ACM ISBN 978-1-4503-8345-5/21/05.

https://doi.org/10.1145/3448018.3458002 and many drivers devote considerable time starting from childhood to develop their skills.

Many studies have reported that exceptional driving skills require correspondingly excellent physiological characteristics [Backman et al. 2005; Jacobs et al. 2002] and brain function/structure characteristics [Bernardi et al. 2014, 2013]. Some studies investigated car racing as a visuomotor task [Land and Tatler 2001; Lappi 2018; Van Leeuwen et al. 2017] and investigated strategies for capturing visual information in terms of gaze and motor coordination. However, few studies noted the importance of attention-level modulation in high-performance driving. In the visual-perceptual feedback loop chain [Mole et al. 2019], attentiveness affects how fast or how well a driver can respond to visual perceptual variables [Posner et al. 1980]. Assessing visual attentiveness during or after driving could contribute to improving driver performance. In this regard, [Rito Lima et al. 2020] observed electroencephalogram (EEG) state changes of a Formula E champion between straight and curve sections; however, whether these changes depend on changes in the driver's attentiveness remained unclear.

In the present study, we focus on spontaneous eyeblink as a promising biometric to reveal the visual and other attention states [Stern et al. 1984] of drivers in-the-wild. Spontaneous eyeblink is a rapid eyelid movement that is distinguishable from both reflexive and voluntary eyeblink in terms of absence of an identifiable eliciting stimulus [Stern et al. 1984]. It normally occurs at a rate of 10-20 $\mathrm{min}^{-1}$, although the individual variance is large [Cruz et al. 2011]. Eyeblink has a direct effect on the visual reaction time. In fact, [Johns et al. 2009] noted that a single eyeblink causes a significant delay in the visual response reaction. [Drew 1951] found that the blink rate varies inversely with the difficulty and amount of control movement necessary in a pencil steering task. Humans blink when implicit and explicit breaks and timings are highly synchronized when viewing a visual story [Nakano et al. 2009]. Further, people show higher blink synchronization when they have higher interest in a video [Nakano and Miyazaki 2019]. Previous studies [Hoppe et al. 2018; Ranti et al. 2020; Shultz et al. 2011] also noted that blinking and/or blink suppression could be a measure of visual attention. In particular, [Hoppe et al. 2018] proposed a computational model that related the probability of blinking and the cost of blinking in terms of task performance.

In this light, we hypothesize that top professional drivers regulate their blink timings effectively to minimize the chance of losing critical information for achieving high driving performance. Further, they show higher and lower blink synchronization for achieving high and low performance because they explicitly or implicitly 
agree when to focus and when to relax, respectively. We investigated this hypothesis by collaborating with a top Japanese racing team and collecting eyeblink data from two drivers in a quasi-racing environment.

\section{METHOD}

\subsection{Subjects}

Drivers A and B, respectively aged 32 and 23 years, participated in this study. Both drove for a top Japanese formula car racing (Super Formula) team. One driver started driving a racing car at the age of five and the other, at the age of six. Further, one has F1 car driving experience and the other, F2 car driving experience.

Before the study, the testing procedure was verbally explained and written informed consent was obtained from both drivers. This study was approved by the ethics committee board of NTT Communication Science Laboratories (no. H30-002), and was conducted in accordance with the Declaration of Helsinki.

\subsection{Apparatus}

We used an image-based technique to collect blink data. The work time required for attaching and removing electrodes for performing electromyography and electrooculography techniques were too long for the drivers' schedule. We used a $120 \mathrm{~Hz}, 400 \times 400$ px binocular eye tracker (Pupil Core, Pupil Labs Gmbh [Kassner et al. 2014]). The world camera of this eye tracker recorded the outward view at $60 \mathrm{~Hz}$. The resulting world camera images were supplementally used to monitor the outward situation. The world camera and two eye trackers were placed on a 3D-printed frame that was then mounted on the edge of the helmet (see Figure $1 \mathrm{~A}$ (left)). Figure 1A (right) shows sample eye images. Image data were collected using a smartphone and processed offline.

Figure 1B shows our data collection system. The car behavior was recorded using a car data logging system (Cosworth Ltd.). The recorded data were exported at a sample rate of $50 \mathrm{~Hz}$. Data were annotated using lap triggers that were triggered by an infrared (IR) transmitter (C16S IR Timing Beacon Receiver, Cosworth Ltd.) on the course. The same IR receiver was used to record the lap information in the eye tracking data. It was installed in the car next to the car behavior lap timing receiver. A microcomputer (PIC16F1705, Microchip Technology Inc.) generated a $1000 \mathrm{~Hz}$ sine wave upon receiving the trigger signal. This sine wave was recorded by our eye-camera-recorder smartphone as a sound signal. This sound was used to synchronize the eye images and the car data logging system.

\subsection{Task procedures}

We were granted permission to perform measurements on the participating drivers during their official free practice (FP) sessions. These sessions are held one day before the qualifying round and final race, and racing teams use them as an opportunity to adjust their car configurations. Typically, racing teams prepare several car configurations that the drivers test through several laps. The fastest lap times in these sessions are often very close to or faster than the fastest qualifying and final lap times. However, during our measurements, because the road condition was wet, the FP lap times were slower than the qualifying and final ones under dry conditions on the next day, and they did not reflect any lack of effort from the drivers in the fastest laps. Having said that, the drivers did drive relatively slowly on some laps, and therefore, we could observe their blinking response on slower laps. We performed our measurements at the Sportsland Sugo track in 2020; both drivers had previously raced on this course.

The drivers wore our eye-camera system and drove with their usual focus on achieving high driving performance and communicating with the team to configure their cars. The weather was slightly rainy. The measurements were conducted between 10:00 am and 12:00 am. We had no control over the drivers' status of drowsiness or fatigue; nonetheless, they were expected to come to the session in perfect condition as would be expected of professional drivers.

\subsection{Data analysis}

In this study, blink onsets were manually annotated using the Pupil Player annotation tool (Pupil Labs Gmbh). The eye video sampling rate was $120 \mathrm{~Hz}$; however, the sampling rate for annotation was limited to $60 \mathrm{~Hz}$ (i.e., 16-ms interval). This was the same as that of the world-side camera. Blink start was defined from the start frame in which the eyelid rapidly moved downward to blink. Therefore, we considered the exact blink onset timings, defined as the starting point when the eyelids move fast, within $16 \mathrm{~ms}$ before the timings we annotated. We assumed that all the blinks were spontaneous during driving because the driver's full-face helmet shielding was enough for preventing reflex blinks.

The extent of within- and inter-person eyeblink synchronization was estimated as described in [Nakano and Miyazaki 2019]. However, it was modified for the measurements performed during formula car driving. Unlike in [Nakano and Miyazaki 2019], we could not assume that drivers' visual events were the same in every lap in terms of temporal structure and contents. Instead, we assumed that the implicitly learned cost for blinking was strongly associated with the track position.

Under this assumption, we calculated the lap distance of blink locations. These were obtained by the linear interpolation from the blink timing data to the car behavior data. Then, we modified it into the lap distance ratio, because the drivers drove subtly different distances in every lap. We used all pairs of laps for within- and inter-subject comparisons. For this purpose, we considered a lap $\mathrm{A}$ and a reference lap B. We converted the blink series $\left\{a_{i}\right\}$ in lap $\mathrm{A}$ and $\left\{b_{i}\right\}$ in lap $\mathrm{B}$ into the lap distance ratio using linear interpolation (Figure 2A). We substituted $a_{i}$ into the blink series $\left\{b_{i}\right\}$ (Figure 2B) and converted $a_{i}$ and $\left\{b_{i}\right\}$ into time units in the opposite way to the last conversion from lap time to lap distance ratio (Figure $2 \mathrm{C}$ ). After this procedure, we can calculate the asynchrony, that is, the difference between blink $a_{i}$ and $\left\{b_{i}\right\}$ in the context of [Nakano and Miyazaki 2019](Figure 2D). In parallel, we prepared 1000 randomized surrogate data of each $\left\{a_{i}\right\}$ and $\left\{b_{i}\right\}$ from the inter-blink interval (IBI) profile of the pair. These randomized data preserved the same IBI but lost the blink order of the original data. By calculating the asynchrony of these randomized data, we could test the null hypothesis that the synchrony of the experimental data happened by chance. We calculated the histogram frequencies of the asynchrony with breaks of $-9.9 \mathrm{~s}$ to $9.9 \mathrm{~s}$ and bin width of 
A

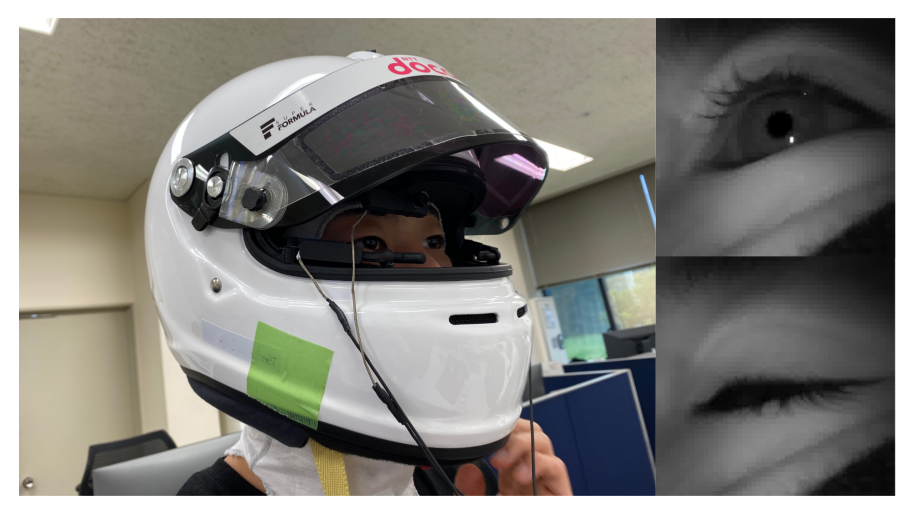

B

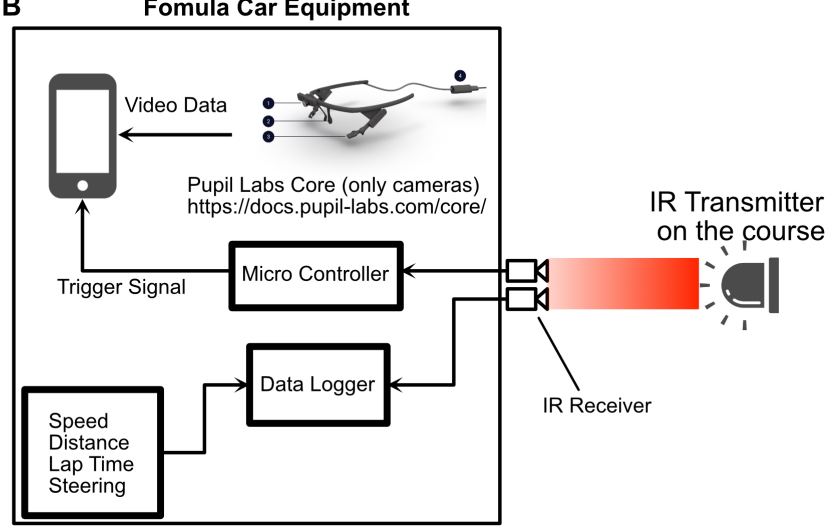

Figure 1: (A) Left: Eye cameras were set inside the full-face helmet using a 3D-printed mount such that they did not impede the driver. Right: Captured open and blinking eye images. (B) The driver wore an eye tracker, and data were collected using a smartphone app. The car data logging system collected the car speed, lap distance, steering angle, and lap time. Both systems were synchronized using aligned photodetectors that were triggered by the IR transmitter on the course.

A
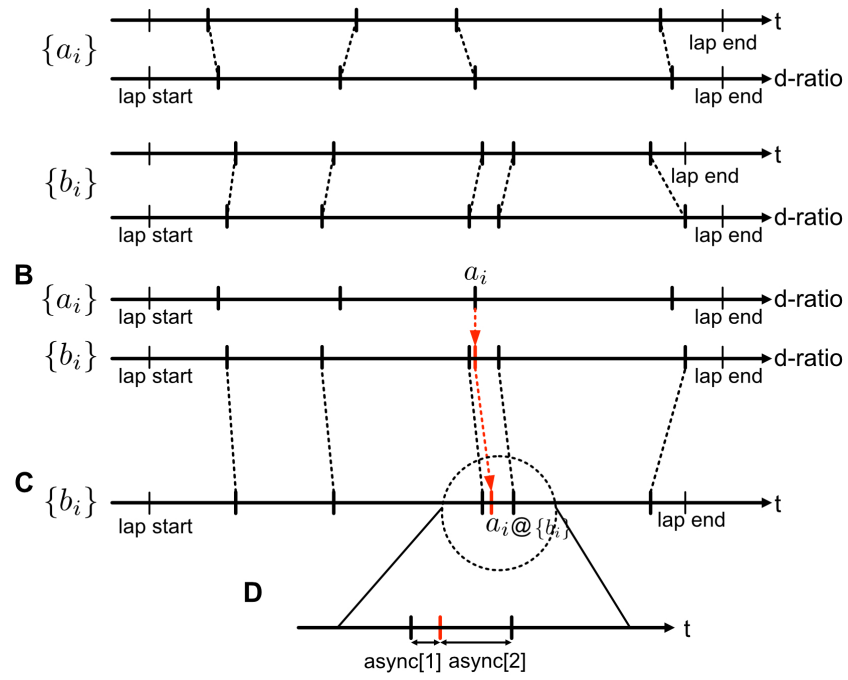

Figure 2: Calculation of asynchrony between two laps that reflects the geographical position in a lap. (A) Conversion from blink timing to blink distance ratio (d-ratio). (B) Inserting a blink event $a_{i}$ into the reference blink events $\left\{b_{i}\right\}$ (C) Reconversion to time unit. (D) Two example calculations of asynchrony between inserted $a_{i}$ and $\left\{b_{i}\right\}$

$0.6 \mathrm{~s}$ and calculated the z-score of the experimental data in each bin using the mean and standard deviation of the randomized data frequency histogram. A z-score of 0 meant that the frequency in the bin could happen by chance. The higher the z-score, the higher was the blink synchronicity among laps.
Table 1: Lap times and run type allocations sorted by lap time.

\begin{tabular}{|c|c|c|c|c|c|c|}
\hline & \multicolumn{3}{|c|}{ Driver A } & \multicolumn{3}{|c|}{ Driver B } \\
\hline & Lap & Lap Time & Run Type & Lap & Lap Time & Run Type \\
\hline 1 & 1 & 97.049 & slow & 1 & 98.578 & slow \\
\hline 2 & 2 & 93.406 & slow & 6 & 94.403 & slow \\
\hline 3 & 6 & 91.692 & slow & 14 & 88.450 & slow \\
\hline 4 & 3 & 86.904 & slow & 2 & 88.410 & slow \\
\hline 5 & 9 & 85.325 & slow & 3 & 85.280 & slow \\
\hline 6 & 4 & 84.847 & medium & 7 & 84.780 & slow \\
\hline 7 & 7 & 84.663 & medium & 9 & 84.610 & slow \\
\hline 8 & 5 & 84.245 & medium & 16 & 84.184 & medium \\
\hline 9 & 8 & 83.764 & medium & 4 & 83.114 & medium \\
\hline 10 & 10 & 83.422 & fast & 19 & 82.804 & medium \\
\hline 11 & 11 & 82.733 & fast & 15 & 82.066 & medium \\
\hline 12 & 12 & 82.683 & fast & 5 & 81.973 & medium \\
\hline 13 & 13 & 80.132 & fast & 11 & 81.643 & medium \\
\hline 14 & 14 & 79.592 & fast & 10 & 81.393 & medium \\
\hline 15 & & & & 8 & 81.106 & fast \\
\hline 16 & & & & 20 & 80.916 & fast \\
\hline 17 & & & & 12 & 80.913 & fast \\
\hline 18 & & & & 13 & 80.590 & fast \\
\hline 19 & & & & 17 & 79.946 & fast \\
\hline 20 & & & & 18 & 79.703 & fast \\
\hline 21 & & & & 21 & 78.701 & fast \\
\hline
\end{tabular}

\section{RESULTS}

\subsection{Blink rate and performance level}

All blink onset locations of Drivers $\mathrm{A}$ and $\mathrm{B}$ and the steering angle from the car data logger are plotted in Figure 3. The mean blink rates of Drivers A and B were $38.5 \mathrm{~min}^{-1}$ (range: $26.2-58.9 \mathrm{~min}^{-1}$ in each lap) and $5.81 \mathrm{~min}^{-1}$ (range: $1.82-15.6 \mathrm{~min}^{-1}$ in each lap). In the following analysis, we normalized the blink rates relative to the total mean blink rate of each driver for this blink rate gap [Nakano and Miyazaki 2019]. We sorted the laps by lap time and divided 

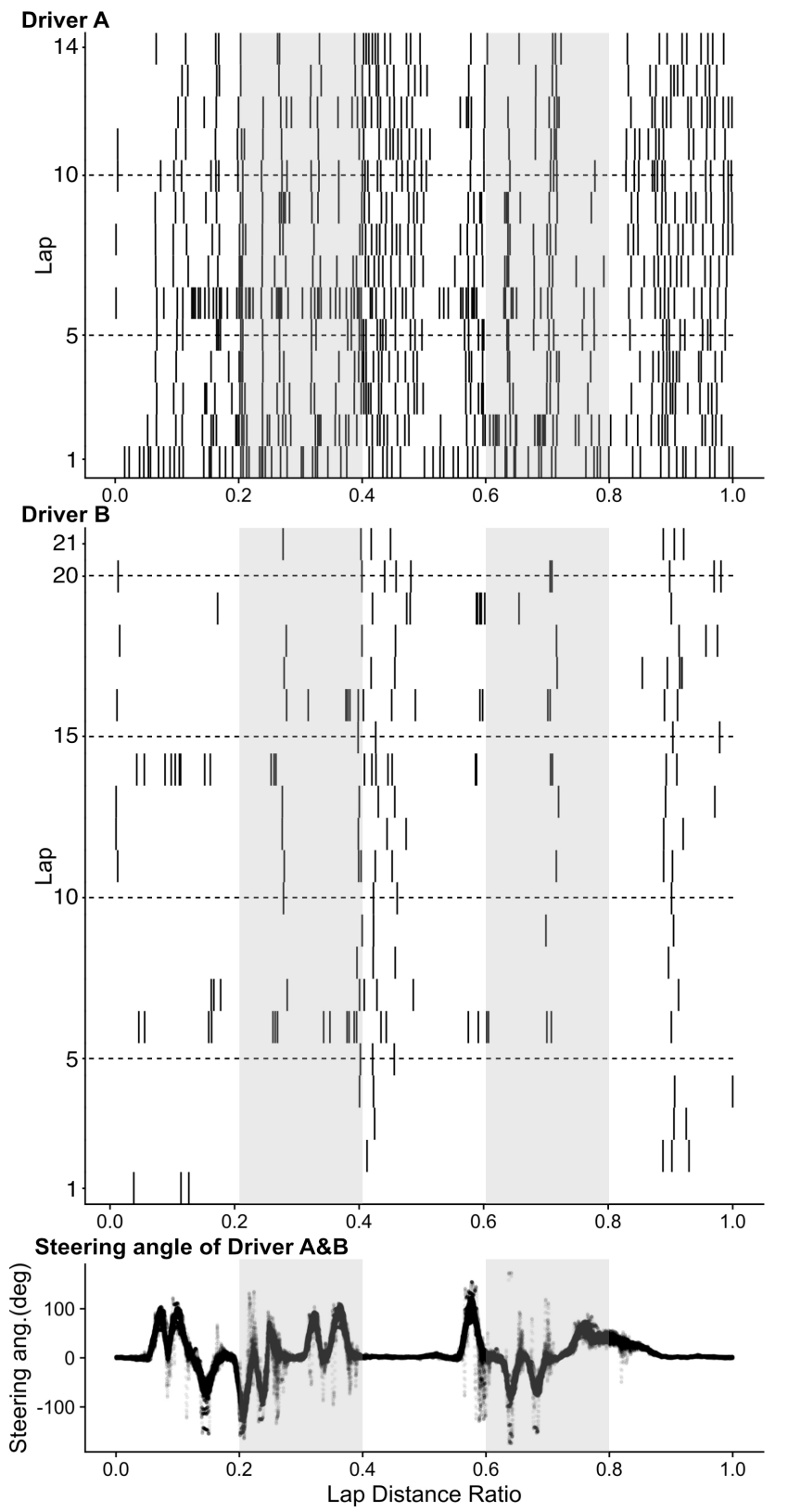

Figure 3: (Top two) Raw blink event location data by lap distance ratio in each lap for Driver $A \& B$. One line represents one blink. (Below) All steering point plots of both drivers. One thin-color dot represents one steering angle at a lap distance ratio.

them into three levels for each driver: slow, medium, and fast. Table 1 shows the breakdown. Qualitatively, blinks are concentrated in the medium and fast laps, and the two main concentration regions (0.4-0.5 and 0.8-1.0 at distance rate) coincide with the beginning of the two less-steering sections.

One of our hypotheses was that drivers suppressed eyeblinks during faster laps. Figure 4 shows the run type vs normalized blink

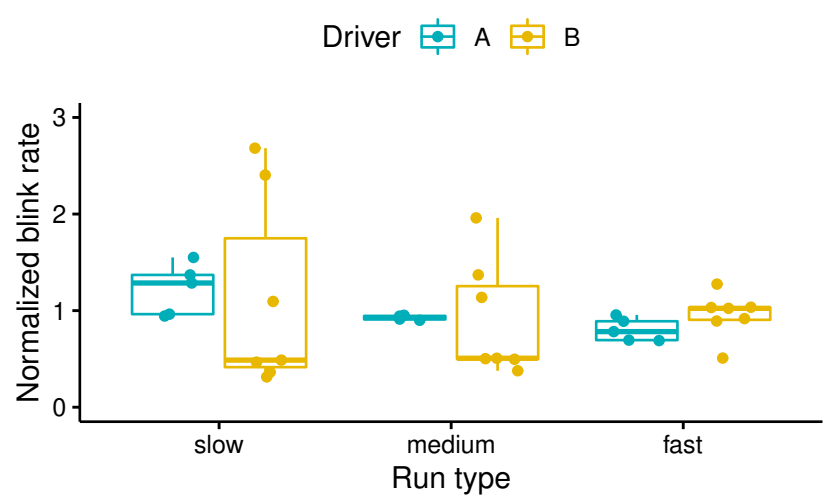

Figure 4: Boxplot of run type vs blink rate in each driver.

rate for each driver. To analyze the effect of run type as a main factor in the normalized blink rate, a one-way analysis of variance (ANOVA) was conducted. It revealed no significant main effect $\left(F_{2,32}=0.934, p=0.403\right)$.

\subsection{Within-driver synchronicity}

Figure 5A shows the $\mathrm{z}$-score asynchrony histograms of each run type for each driver. For both drivers, the histograms showed an evident peak in the bin $0 \mathrm{~ms} \pm 300 \mathrm{~ms}$. The mean $\mathrm{z}$-value in the bin around $0 \mathrm{~s}$ across all laps for both drivers was significantly higher than 0 ( $t$-test, $\left.t_{601}=30.935, p<0.001\right)$.

Figure $5 \mathrm{~B}$ shows the run type vs $\mathrm{z}$-score in the $0 \mathrm{~ms} \pm 300 \mathrm{~ms}$ bin. The two-way ANOVA with the z-score as response and with drivers and run types as factors revealed no significant interaction of run type and driver $\left(F_{2,172}=1.148, p=0.320\right)$, a significant main effect of run type $\left(F_{2,172}=15.942, p<0.001\right)$, and a significant main effect of driver $\left(F_{1,172}=5.756, p=0.017\right)$. A post-hoc pairwise $\mathrm{t}$-test with Bonferroni correction revealed the differences between run types: slow vs medium ( $p<0.001)$, slow vs fast $(p<0.001)$, and medium vs fast $(p=1.00)$. Both drivers showed higher synchronization in the fast and medium laps than in the slow laps, and Driver A showed higher synchronization than Driver B did.

\subsection{Across-driver synchronicity}

To examine the synchronization between the drivers, we calculated the asynchrony from Driver A's laps to Driver B's laps and viceversa. The whole mean of the $\mathrm{z}$-score around $0 \mathrm{~s}$ was significantly higher than $0\left(t_{587}=31.453, p<0.001\right)$. This indicates that both drivers generally blinked at similar locations in all run types.

Then, we examined the effect of the driving performance on the blink synchronization between the drivers. We tested every combination of laps from Drivers A and B in each run type for calculating the synchronicity. These values are plotted in Figure $6 \mathrm{~A}$. Only the $\mathrm{z}$-values in the $0 \mathrm{~ms} \pm 300 \mathrm{~ms}$ bin in each run type are extracted and plotted in Figure 6B. Analyzing the mean blink synchronization around $0 \mathrm{~ms}$ as the response by a one-way ANOVA with the run type as a factor revealed a significant main effect of run type $\left(F_{2,193}=22.491, p<0.001\right)$. A post-hoc pairwise t-test with Bonferroni correction revealed the differences between run 

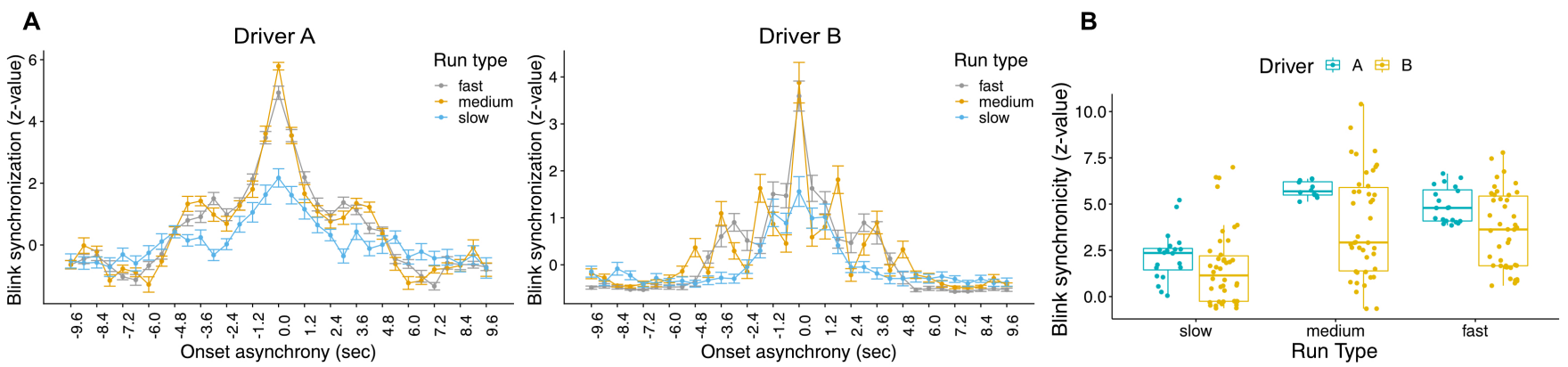

Figure 5: Distributions of within-driver synchronicity.
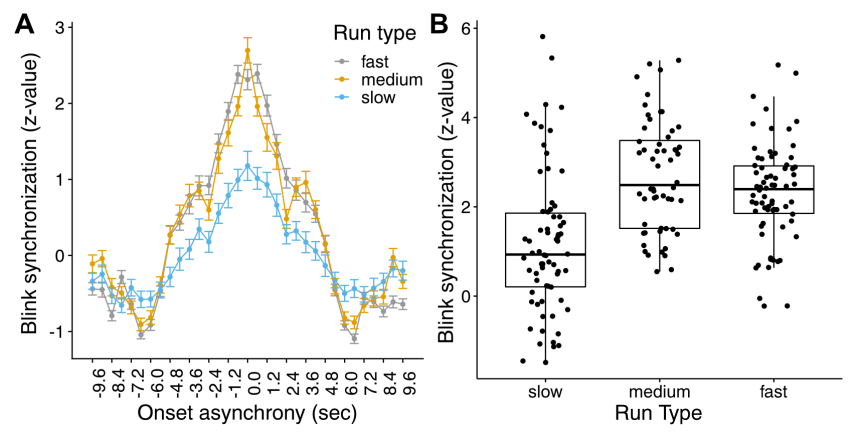

Figure 6: Distributions of across-driver synchronicity.

type: fast vs slow $(p<0.001)$, medium vs slow $(p<0.001)$, and fast vs medium ( $p=0.335$ ). This result indicated that medium and fast performance laps showed higher synchronization across drivers than slow laps did; however, no significant difference was seen for medium vs fast laps.

\section{DISCUSSION}

In the present measurement, we were concerned with spontaneous eyeblink during formula car driving as a measure of cognitive state variables [Stern et al. 1984]. The averaged blink rates of the two drivers were largely different $\left(38.5 \mathrm{~min}^{-1}\right.$ and $\left.5.81 \mathrm{~min}^{-1}\right)$. Considering that a blink suppresses visual awareness for around 200-400 ms [Volkmann et al. 1980], these blink rates might correspond to a loss of visual information for $\simeq 8 \mathrm{~s}(14 \%)$ and $1.3 \mathrm{~s}(2.1 \%)$ per minute in one lap, respectively. This loss of visual information could be critical during race driving at a maximum speed exceeding 250 $\mathrm{km} / \mathrm{h}$ (average speed of $150 \mathrm{~km} / \mathrm{h}$ on this track). Therefore, the eyeblink behavior should impact the performance.

Among the eyeblink indices investigated in this study, the normalized blink frequency change did not show a significant difference with the change in driving performance (Figure 4). In contrast, the synchronicity of eyeblink based on geometric location increased significantly as the driving performance increased (Figure 5). This result suggests that the blink pattern on the track is related to the driving performance. To achieve a fast lap-time, drivers have to manipulate the throttle, brakes, gear shifts, and steering precisely at the right moment on the track [Van Leeuwen et al. 2017]. Toward this end, visual attention might be modulated during the lap so as to capture or not lose critical visual information for controlling the car, especially during high-performance laps. As previous studies have suggested [Hoppe et al. 2018], this modulation of visual attention could be reflected in the blink patterns.

Further, high blink synchronicity across drivers was observed in higher-performance laps (Figure 6) even though the blink rates were quite different between the drivers. These results support our hypothesis that the modulation pattern of visual attention in a high-performance lap is similar between the drivers. As mentioned above, the blink led to the drivers losing the visual information, and the amount of loss differed between the drivers. In contrast, the similarity of blink patterns between the drivers suggests that the timing of visual information loss is similar. This might indicate that both drivers have similar control patterns for their visual attention level on the track. This might be a special skill that is characteristic of top-level drivers. However, we could not verify this from our observations alone because we cannot examine whether normal drivers show similar blink patterns when they drive the same formula car, and because our sample size of elite drivers is as small as two. These are the major limitations of our study.

We suggest that one might be able to access the fast modulation pattern of visual attentiveness, which is related to sports performance in in-the-wild situations, through the blink synchronization metric. We observed the eye blinks in a real racing situation that contained much measurement noise such as large sounds, high oscillations, wide range of lighting environments, and rainy conditions. Even under such severe conditions for measuring biometrics, blinks could be obtained robustly. We suggest that our analysis may provide a clue to estimate how much weight humans give to external visual stimuli during a task outside the laboratory with good temporal resolution. Such information could be useful for evaluating sports players, improving traffic safety, and efficient camera control for driving automated vehicles.

\section{REFERENCES}

Jani Backman, Keijo Häkkinen, Jari Ylinen, Arja Häkkinen, and Heikki Kyröläinen. 2005. Neuromuscular performance characteristics of open-wheel and rally drivers. fournal of strength and conditioning research 19, 4 (2005), 777.

Giulio Bernardi, Luca Cecchetti, Giacomo Handjaras, Lorenzo Sani, Anna Gaglianese, Riccardo Ceccarelli, Ferdinando Franzoni, Fabio Galetta, Gino Santoro, Rainer Goebel, et al. 2014. It's not all in your car: functional and structural correlates of exceptional driving skills in professional racers. Frontiers in human neuroscience 8 (2014), 888. 
Giulio Bernardi, Emiliano Ricciardi, Lorenzo Sani, Anna Gaglianese, Alessandra Papasogli, Riccardo Ceccarelli, Ferdinando Franzoni, Fabio Galetta, Gino Santoro, Rainer Goebel, et al. 2013. How skill expertise shapes the brain functional architecture: an fMRI study of visuo-spatial and motor processing in professional racing-car and naïve drivers. PloS one 8, 10 (2013), e77764.

Antonio A.V. Cruz, Denny M. Garcia, Carolina T. Pinto, and Sheila P. Cechetti. 2011 Spontaneous Eyeblink Activity. The Ocular Surface 9, 1 (2011), 29 - 41. https: //doi.org/10.1016/S1542-0124(11)70007-6

George Charles Drew. 1951. Variations in reflex blink-rate during visual-motor tasks. Quarterly fournal of Experimental Psychology 3, 2 (1951), 73-88.

David Hoppe, Stefan Helfmann, and Constantin A. Rothkopf. 2018. Humans quickly learn to blink strategically in response to environmental task demands. Proceedings of the National Academy of Sciences of the United States of America 115, 9 (2018), 2246-2251. https://doi.org/10.1073/pnas.1714220115

Patrick L. Jacobs, Stephen E. Olvey, Brad M. Johnson, and Kelly A. Cohn. 2002. Physiological responses to high-speed, open-wheel racecar driving. Medicine and Science in Sports and Exercise 34, 12 (2002), 2085-2090. https://doi.org/10.1097/00005768200212000-00033

Murray Johns, Kate Crowley, Robert Chapman, Andrew Tucker, and Christopher Hocking. 2009. The effect of blinks and saccadic eye movements on visual reaction times. Attention, Perception, \& Psychophysics 71, 4 (2009), 783-788.

Moritz Kassner, William Patera, and Andreas Bulling. 2014. Pupil: An Open Source Platform for Pervasive Eye Tracking and Mobile Gaze-based Interaction. In $\mathrm{Ad}$ junct Proceedings of the 2014 ACM International foint Conference on Pervasive and Ubiquitous Computing (Seattle, Washington) (UbiComp '14 Adjunct). ACM, New York, NY, USA, 1151-1160. https://doi.org/10.1145/2638728.2641695

Michael F Land and Benjamin W Tatler. 2001. Steering with the head: The visual strategy of a racing driver. Current Biology 11, 15 (2001), 1215-1220.

Otto Lappi. 2018. The Racer's Mind-How Core Perceptual-Cognitive Expertise Is Reflected in Deliberate Practice Procedures in Professional Motorsport. Frontiers in psychology 9 (2018), 1294.
Callum D Mole, Otto Lappi, Oscar Giles, Gustav Markkula, Franck Mars, and Richard M Wilkie. 2019. Getting back into the loop: the perceptual-motor determinants of successful transitions out of automated driving. Human factors 61, 7 (2019), 10371065.

Tamami Nakano and Yuta Miyazaki. 2019. Blink synchronization is an indicator of interest while viewing videos. International fournal of Psychophysiology 135 (2019), 1 - 11. https://doi.org/10.1016/j.ijpsycho.2018.10.012

Tamami Nakano, Yoshiharu Yamamoto, Keiichi Kitajo, Toshimitsu Takahashi, and Shigeru Kitazawa. 2009. Synchronization of spontaneous eyeblinks while viewing video stories. Proceedings of the Royal Society B: Biological Sciences 276, 1673 (2009), 3635-3644.

Michael I Posner, Charles R Snyder, and Brian J Davidson. 1980. Attention and the detection of signals. Journal of experimental psychology: General 109, 2 (1980), 160.

Carolyn Ranti, Warren Jones, Ami Klin, and Sarah Shultz. 2020. Blink Rate pattern provide a Reliable Measure of individual engagement with Scene content. Scientific Reports (2020).

Ines Rito Lima, Shlomi Haar, Lucas Di Grassi, and A. Aldo Faisal. 2020. Neurobehavioural signatures in race car driving: a case study. Scientific Reports 10, 1 (2020), 1-9. https://doi.org/10.1038/s41598-020-68423-2

Sarah Shultz, Ami Klin, and Warren Jones. 2011. Inhibition of eye blinking reveals subjective perceptions of stimulus salience. Proceedings of the National Academy of Sciences of the United States of America 108, 52 (2011), 21270-21275. https: //doi.org/10.1073/pnas.1109304108

John A Stern, Larry C Walrath, and Robert Goldstein. 1984. The endogenous eyeblink. Psychophysiology 21, 1 (1984), 22-33.

Peter M Van Leeuwen, Stefan de Groot, Riender Happee, and Joost CF de Winter. 2017. Differences between racing and non-racing drivers: A simulator study using eye-tracking. PLoS one 12, 11 (2017), e0186871.

Frances C Volkmann, Lorrin A Riggs, and Robert K Moore. 1980. Eyeblinks and visua suppression. Science 207, 4433 (1980), 900-902. 